\title{
EXTINCTION AND TRANSFORMATION: An Abstract
}

\author{
Andrew T. Young \\ Astronomy Department, San Diego State University \\ San Diego, California 92182-0334
}

The basic principles of heterochromatic extinction show that the approach used in the visible should not work well in the infrared, where molecular line absorption rather than continuous scattering dominates the extinction. Not only does this extinction change very rapidly with wavelength (so that stellar color becomes only weakly correlated with effective extinction), but also many of the lines are saturated (so that Forbe's curve-of-growth effect is much more severe in the IR.) Furthermore, broadband IR colors are more undersampled than those in the visible, so aliasing errors make them correlate even less with extinction.

Reduction to outside the atmosphere is difficult, but the rational approximation

$$
\Delta m=\frac{\left(A M^{2}+B M+C\right)}{\left(M+M_{0}\right)}
$$

models the extinction quite well as a function of air mass, $M$. As $M_{0}$ is nearly indeterminate from observations over the accessible range of air masses, it can be guessed better than it can be found from observations. Good guesses for $M_{0}$, based on the synthetic photometry of Manduca and Bell (1979) from $M=0$ to 3, give extra-atmospheric magnitudes extrapolated from the range $M=1.0$ to 2.5 that are accurate to a few hundredths of a magnitude. Even if one assumes $M_{0}=1.0$ in every case, the largest extrapolation errors are still about three times smaller than those from linear fits (see Table III of Manduca and Bell, 1979).

Extinction can be well determined with a few stars per hour if the observations are carefully planned (Young, 1974). $M$ should not exceed 3 , beyond which its values are uncertain owing to the variable scale height of water vapor. As in the visible, the determination of nightly extinction can be strengthened enormously by reducing several nights together (Young and Irvine, 1967; Manfroid and Heck, 1983).

To prevent aliasing time-dependent extinction into the fitted parameters, one must observe both rising and setting stars and solve for time-dependent parameters, as in Rufener's (1964) "M and D"method. This is more urgent in the infrared than in the visible, because changes in water vapor can cause large effects on the IR extinction without producing an obvious visible effect. Furthermore, because the water vapor that dominates the IR extinction has a smaller scale height than the atmosphere as a whole, one must use an air mass formula that takes this into account (as well as allowing for the distinction between true and refracted zenith distances).

The only solution to the transformation problem is to satisfy the sampling theorem, which may be difficult in the IR because of gaps due to saturated telluric absorptions. 


\section{REFERENCES}

Forbes, J.D. 1842, Phil. Trans. 132, 225-273

Manduca, A., and Bell, R.A. 1979, Pub. A.S.P.91, 848

Manfroid, J., and Heck, A. 1983, Astron. Astrophys.120, 302-306

Rufener, F. 1964, Pub. Obs. Genève, Série A, Fasc. 66

Young, A.T., and Irvine, W.M. 1967, Astron. J.72, 945-950

Young, A.T. 1974, in Methods of Experimental Physics, Vol. 12 (Astrophysics, Part. A: Optical and Infrared), ed. by N. Carleton (Academic Press, New York) pp. 123-192 